\author{
A. GALLER \\ T. FEURER
}

\title{
Pulse shaper assisted short laser pulse characterization
}

\author{
Institute of Applied Physics, University of Bern, Sidlerstr. 5, 3012 Bern, Switzerland
}

\begin{abstract}
Received: 10 July 2007/Revised version: 13 November 2007 Published online: 29 January 2008 • (C) Springer-Verlag 2008

ABSTRACT We demonstrate that a pulse shaper is able to simultaneously act as an optical waveform generator and a short pulse characterization device when combined with an appropriate nonlinear element. We present autocorrelation measurements and their frequency resolved counterparts. We show that control over the carrier envelope phase allows continuous tuning between an intensity-like and an interferometric autocorrelation. By changing the transfer function other measurement techniques, for example STRUT, are easily realized without any modification of the optical setup.
\end{abstract}

PACS 42.65.Re; 42.30.Lr; 42.30.Rx

\section{$1 \quad$ Introduction}

Recent decades have seen a rapid evolution in the characterization of short laser pulses. A number of techniques, such as frequency resolved optical gating (FROG) [1], spectral phase interferometry for direct electric-field reconstruction (SPIDER) [2], or spectrally and temporally resolved up-conversion techniques (STRUT) [3] etc., yield experimental results from which the complete electric field can be reconstructed. Simultaneously, our ability to shape light fields in a controlled way has dramatically increased [4]. Pulse shaping has come to the point where not only the phase and/or amplitude of a single polarization component can be manipulated but also the vectorial nature of the field [5], or even the space time distribution of the field [6]. Still, in most experiments the shaping of light pulses and their characterization is performed in two separate optical arrangements. Only a few experiments have been reported where the pulse shaper was used as an integral part of the diagnostic setup, i.e. in the multiphoton intrapulse interference phase scan method [7], in the shaper-assisted collinear SPIDER [8], and in time-domain interferometry with an acousto-optic modulator [9]. Here, we show that a pulse shaper may be used to mimic most characterization arrangements and only a suitable nonlinear element is required to perform the corresponding measurements. This is convenient as the nonlinear element, for example a nonlinear crystal or a two-photon diode, can be placed exactly at

E-mail: andreas.galler@iap.unibe.ch the position of the experiment itself requiring little space and, thus, allowing characterization of waveforms exactly where needed. In addition, the unique capability to control the carrier envelope phase can be used to reduce the number of required sample points in interferometric measurements.

\section{$2 \quad$ Experimental}

Our main objective is to replace a standard short pulse characterization setup by a single nonlinear element and to use a pulse shaping apparatus, first, to create the desired shaped waveform and, second, to produce two replica of the shaped waveform and to scan the delay between them. Both operations are linear and can be performed simultaneously by the same device.

A schematic of the experimental setup is shown in Fig. 1. The laser pulses from a Ti:sapphire oscillator (KM Labs) were directed to a pulse shaper in a standard $4 f$ zero dispersion geometry. The center wavelength of the pulses was $820 \mathrm{~nm}$, the spectral bandwidth was approximately $60 \mathrm{~nm}$, and the repetition rate was $90 \mathrm{MHz}$. The spatial light modulator (JenOptik SLM 640-d) in the Fourier plane of the zero dispersion compressor has 640 pixel and can modulate the amplitude as well as the phase of each spectral component. The shaper's output was then guided to the experiment, which for characterization purposes was replaced by a suitable combination of a nonlinear element and a linear detector. While the scanning second order autocorrelation measurements were performed with a nonlinear diode (SiC) [10], the FROG and STRUT measurements used a nonlinear crystal (BBO) and the nonlinear response was recorded with a spectrometer. We would like to stress that the two replica are inherently parallel, which under normal circumstances would lead to an interferometric measurement.

\section{Scanning second order autocorrelation}

Scanning autocorrelation measurements require two identical replicas of the pulse to be characterized which are subsequently directed to a nonlinear medium whose nonlinear response is measured as a function of the delay between the replica. Utilizing the pulse shaper's ability to control the spectral amplitude and/or phase of the original pulse, the two replica can easily be generated. Moreover, scanning the delay between the two replica can be realized, first, by shifting 


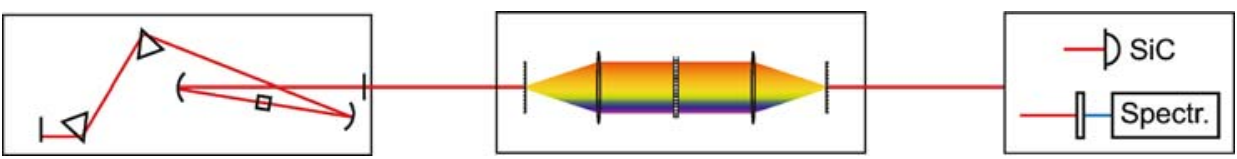

FIGURE 1 Schematic of the experimental setup

simultaneously the envelope and the carrier phase as it is the case for a mechanical delay line or, second, by shifting only the envelope and keeping the carrier phase fixed. Even any intermediate carrier envelope phase shift between the two extremes is possible. The waveform consisting of two replica of the originally Fourier-limited or shaped pulse $E_{0}(t)$ is

$$
\begin{aligned}
E(t)= & E_{0}(t+\tau / 2) \exp \left[\mathrm{i} \omega_{\mathrm{c}}(t+\gamma \tau / 2)\right] \\
& +E_{0}(t-\tau / 2) \exp \left[\mathrm{i} \omega_{\mathrm{c}}(t-\gamma \tau / 2)\right],
\end{aligned}
$$

where $\omega_{\mathrm{c}}$ is the carrier frequency and $\tau$ is the delay between the two replica which are symmetric with respect to $t=0$. The carrier phase remains unchanged for $\gamma=0$ and shifts together with the envelope for $\gamma=1$. The spectral transfer function to produce such two replica is easily found as

$$
\begin{aligned}
M(\omega) & =\frac{1}{2}\left[\mathrm{e}^{\mathrm{i}\left[\omega-(1-\gamma) \omega_{\mathrm{c}}\right] \tau / 2}+\mathrm{e}^{-\mathrm{i}\left[\omega-(1-\gamma) \omega_{\mathrm{c}}\right] \tau / 2}\right] \\
& =\cos \left[\left(\omega-(1-\gamma) \omega_{\mathrm{c}}\right) \frac{\tau}{2}\right] .
\end{aligned}
$$

Pixelated devices usually have gaps separating two neighboring pixels. Those spectral components passing through the gaps remain unmodulated and cause an unwanted replica at $t=0$ with a relative amplitude that is determined by the gap/pixel ratio, typically a few percent [11]. Nonetheless, the $t=0$ replica may perturb the measured autocorrelation trace. The problem can be avoided if only one replica is scanned and the other remains fixed at $t=0$. In this case, the transfer function is

$M(\omega)=\frac{1}{2}\left[1+\mathrm{e}^{-\mathrm{i}\left[\omega-(1-\gamma) \omega_{\mathrm{c}}\right] \tau}\right]$.

Besides the gap replica there are other possible waveform distortions. First, the limited time window of the shaper and the decreasing amplitude of the two replica associated with it as they are shifted away from time zero. Second, the pixel replica and, third, the wrap replica [11]. For the setup presented here and for a maximum delay of $\pm 1 \mathrm{ps}$, none of these systematic errors cause any major problems. Within this delay range the amplitude varies by only $2 \%$, pixel replicas contribute less than $0.01 \%$ to the signal, and no wrap replica appear if the shaper is used as a characterization tool only. The output waveform of the pulse shaper, i.e. the two collinear replica, is then focused onto a $\mathrm{SiC}$ diode whose nonlinear response is measured.

Figure 2a-c show a sequence of autocorrelation traces for $\gamma=0,0.5$ and 1 , respectively, using the transfer function (2). All traces have been recorded with unshaped laser pulses and have a contrast ratio of $8: 1$ which is characteristic for such a measurement. When the carrier phase remains fixed and only the two envelopes are shifted $(\gamma=0)$, the measured autocorrelation trace in Fig. 2a shows no oscillations. This may seem surprising given the fact that the two replica propagate collinearly, but beautifully demonstrates the ability to control the carrier envelope phase. The measured autocorrelation is identical to the upper envelope of the interferometric second order autocorrelation. For $\gamma>0$ oscillations appear and their periodicity can be adjusted at will through a judicious choice of $\gamma$. While for $\gamma=1$ the oscillation frequency matches the carrier frequency, for $\gamma=0.5$ the oscillation frequency is reduced by a factor of two. The corresponding Fourier transforms in Fig. 2e-g indicate that next to the expected contributions at $\gamma \omega_{\mathrm{c}}$ and $2 \gamma \omega_{\mathrm{c}}$ additional peaks appear. As discussed above, these are due to the gap replica at $t=0$ and can be avoided if only one of the two replica is scanned, by using the transfer function (3), as demonstrated in Fig. 2d. The corresponding Fourier transform in Fig. 2h confirms the absence of the intermediate frequency peaks.

\section{$4 \quad$ Frequency resolved autocorrelation}

If the $\mathrm{SiC}$ diode is replaced by a $\mathrm{BBO}$ crystal and the second harmonic signal is spectrally resolved, then the

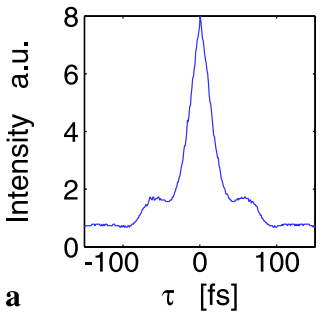

a

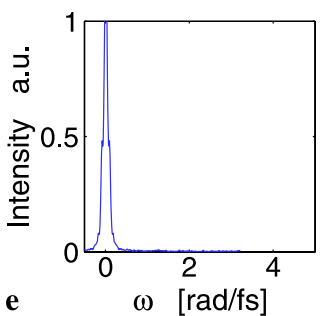

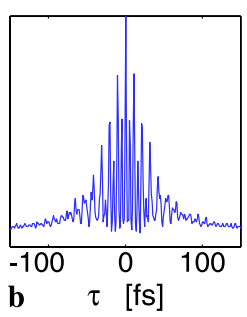

b

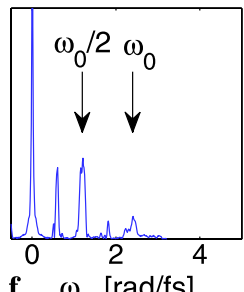

f $\omega[\mathrm{rad} / \mathrm{fs}]$
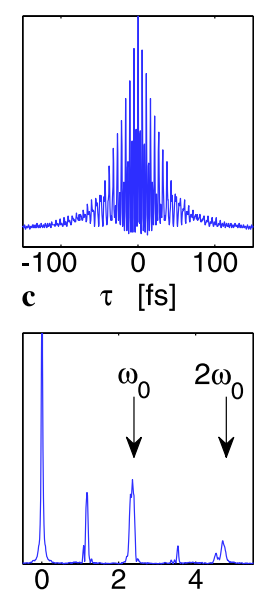

g $\omega[\mathrm{rad} / \mathrm{fs}]$

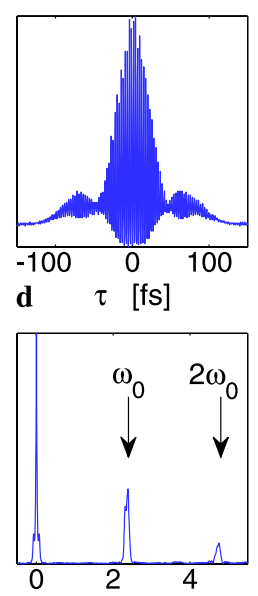

h $\quad \omega[\mathrm{rad} / \mathrm{fs}]$
FIGURE 2 Measured autocorrelation traces for $\mathbf{a} \gamma=0, \mathbf{b} \gamma=0.5$, and $\mathbf{c} \gamma=1$ using (2). d Autocorrelation trace for $\gamma=1$ using (3). The corresponding Fourier transforms are shown in $\mathbf{e}-\mathbf{h}$ 


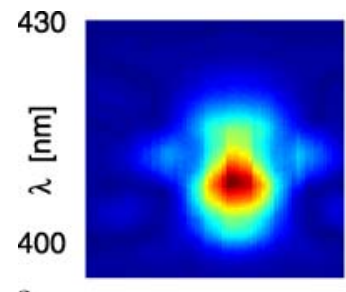

a

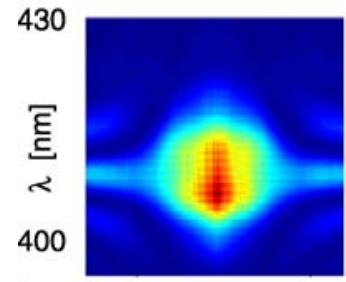

b

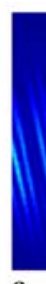

c

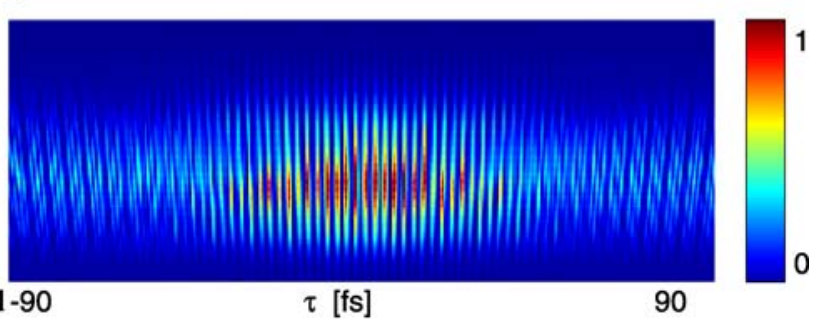

FIGURE 3 a Standard FROG trace for reference. Measured time frequency distributions for $\mathbf{b} \gamma=0, \mathbf{c} \gamma=0.5$, and $\mathbf{d} \gamma=1$

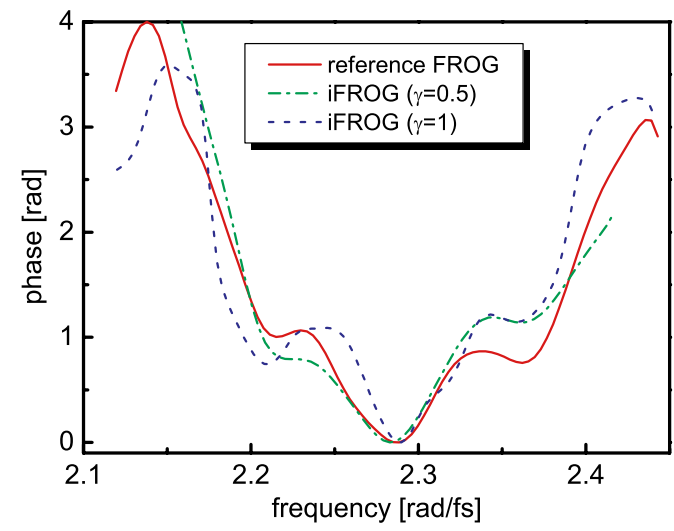

FIGURE 4 Phase retrieved from the measurements in Fig. 3a, c, and d

measurement produces a time frequency distribution which is somewhat reminiscent of a spectrogram. Again, by specifying $\gamma$ the periodicity of the interference fringes can be adjusted at will. Figure 3 shows a sequence of four time frequency distributions of the original laser pulse, where Fig. 3a depicts a standard FROG trace for reference and Fig. 3b-d correspond to $\gamma$ equal to $0,0.5$, and 1 , respectively.

As expected, the measured time-frequency distribution in Fig. $3 b$ shows no oscillations $(\gamma=0)$, however, it differs from the standard FROG trace because of the collinear setup. For $\gamma=1$ we find a distribution equal to the recently published iFROG $[12,13]$. As soon as the information is encoded on the interference fringes the standard FROG trace can be extracted through appropriate processing of the measured data $[12,13]$. This comes at the expense of a much higher sampling rate as the carrier frequency must be sampled with a minimum resolution given by the Nyquist limit. However, with the ability to control the carrier envelope phase and, thus, the fringe spacing, the sampling requirement can be mitigated; for example, when setting $\gamma=0.5$, as shown in Fig. 3c, the required sampling rate reduces by a factor of two. The minimum $\gamma$ allowed is roughly determined by the spectral bandwidth of the original pulse. The phases extracted from the reference FROG trace and from the measurements in Fig. $3 \mathrm{c}$ and d are compared in Fig. 4 and agree well with each other.

Next, the laser pulses were sent through a $50 \mathrm{~mm}$ thick piece of fused silica glass and were analyzed through a refer- ence FROG trace and the shaper-assisted measurement with $\gamma=1$. The results are shown in Fig. 5a and b. From Fig. 5b the standard FROG trace can be recovered through appropriate Fourier processing and the result is shown in Fig. 5c. The phases extracted from both traces are compared to the expected phase modulation in Fig. 5d; both measurements agree well with the simulation.

\section{5}

\section{STRUT}

An alternative but because of signal-to-noise problems rarely used measurement technique is STRUT [3]. Briefly, a narrow spectral slice of the original spectrum is selected through appropriate amplitude filtering and timecorrelated with a copy of the original pulse (reference pulse). A time frequency distribution is obtained when the nonlinear correlation measurement is repeated for increasing center wavelengths of the spectral slice. The maximum of the nonlinear correlation is observed at a delay where the spectral slice temporally overlaps with the corresponding portion of the reference spectrum. That is, the spectral phase $\varphi(\omega)$ of the original pulse can be readily extracted, because the delay $\tau_{\max }(\omega)$ where the maximum signal appears is determined by $\partial \varphi(\omega) / \partial \omega$. Contrary to the FROG measurement, there is no time ambiguity and the STRUT trace is generally asymmetric with respect to time. In order to realize a shaper assisted STRUT measurement the appropriate transfer function must be found,

$$
M(\omega)= \begin{cases}A & \omega<\omega_{0}-\frac{\Delta \omega}{2} \vee \omega>\omega_{0}+\frac{\Delta \omega}{2} \\ A+(1-A) \mathrm{e}^{\mathrm{i} \omega \tau} & \omega_{0}-\frac{\Delta \omega}{2} \leq \omega \leq \omega_{0}+\frac{\Delta \omega}{2}\end{cases}
$$

where $\tau$ is the delay between the spectral slice and the reference pulse and $\omega_{0}$ is the position and $\Delta \omega$ the width of the spectral slice. To match the relative intensities of the spectral slice and the time delayed reference pulse the relative amplitude $A$ of the latter is lowered to 0.2. We would like to emphasize, that the experimental setup is exactly the same as in the previous section, only the transfer function was adjusted when switching from one measurement technique to the other. Because the two pulses are collinear the measured time frequency distribution will be an interferometric version of the 

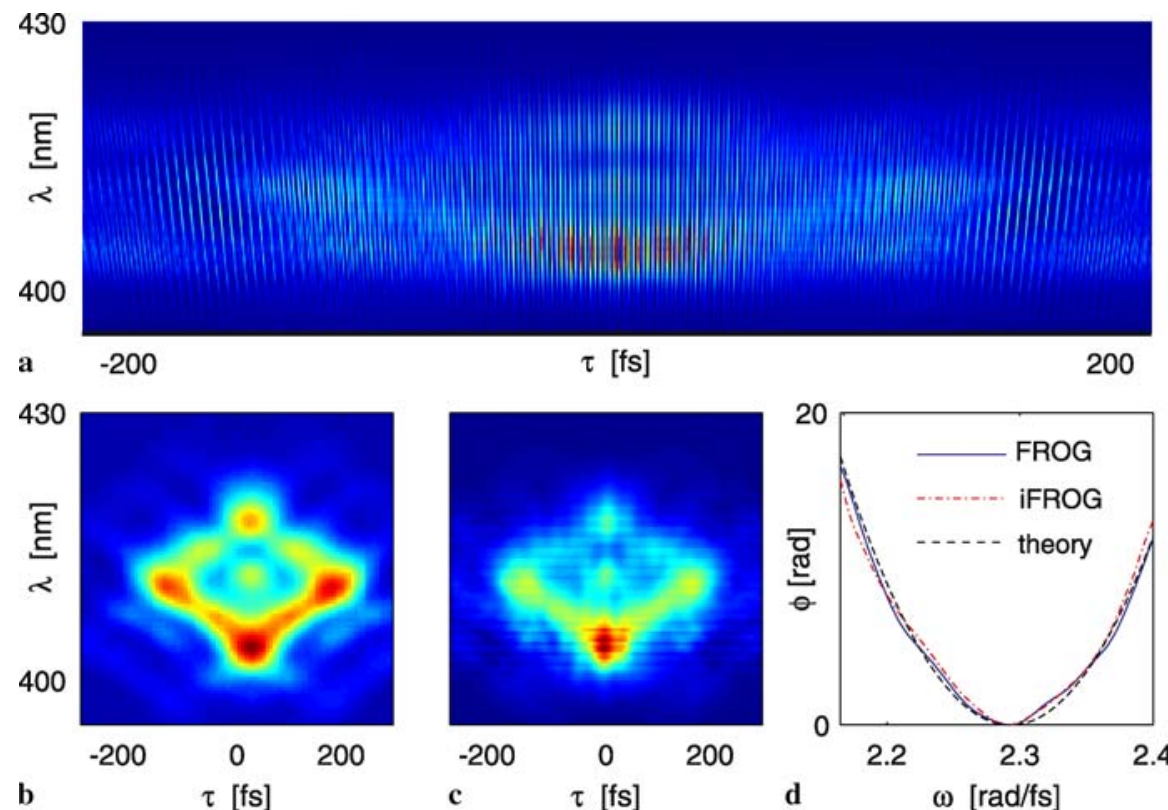

FIGURE 5 a iFROG measurement and b Reference FROG. c FROG trace retrieved from $\mathbf{b}$. d Extracted phases from the FROG and iFROG traces of a laser pulse that passed through $5 \mathrm{~cm}$ of fused silica

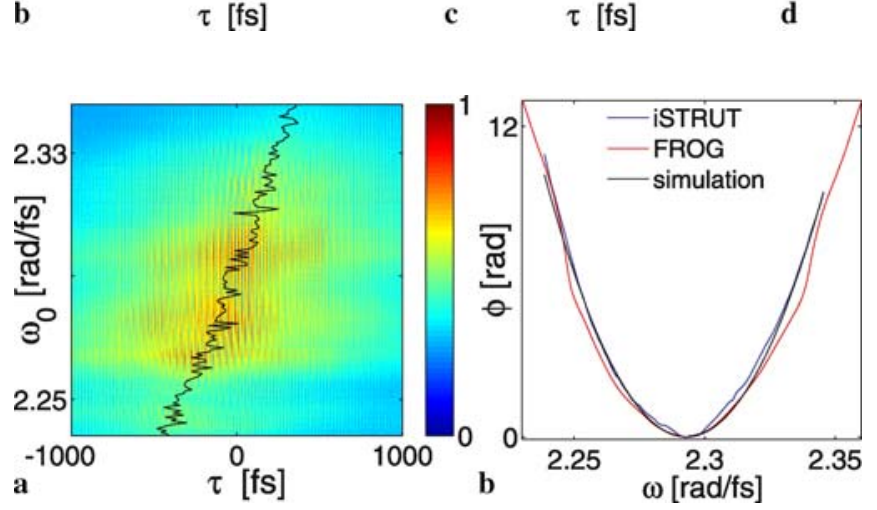

FIGURE 6 a Shaper assisted STRUT measurement of a laser pulse with a GVD of $3000 \mathrm{fs}^{2}$. The position of the maximum correlation signal is indicated by the black line. b Comparison between the extracted phase and the phase retrieved from a reference FROG

standard STRUT trace, henceforth denoted as iSTRUT. The standard STRUT trace is easily extracted from the iSTRUT by Fourier transforming the measured distribution with respect to the time axis and by selecting only the frequency components around the frequency origin. The procedure is very much alike the one used for the iFROG. An example of a shaper assisted iSTRUT measurement is shown in Fig. 6a.

Here, the shaper has been used to impose a quadratic phase modulation of $3000 \mathrm{fs}^{2}$ onto the pulse and, at the same time, to perform the iSTRUT measurement. The black line in Fig. $6 \mathrm{a}$ indicates the position of the maxima in the correlation signal and the almost linear dependence hints at a mostly quadratic phase. The extracted phase is shown in Fig. 6b and corresponds well to the phase retrieved from a reference FROG measurement and the phase written to the shaper. While the sign of the phase from the FROG trace is usually based on reasonable assumptions, there is no time ambiguity in the iSTRUT measurement. Furthermore, the iSTRUT, as for the iFROG, is self-calibrated because the carrier frequency can be deduced from the fringe period or vice versa.

\section{Conclusion}

We have shown that a pulse shaper is able to simultaneously act as an optical waveform generator and a measurement device when combined with an appropriate nonlinear element and a detector. A simple second order autocorrelation measurement was realized, demonstrating artefacts due to unwanted replica and ways to avoid them. Control over the carrier envelope phase allowed to continuously tune between an intensity-like and an interferometric autocorrelation. The frequency resolved autocorrelation was shown to be identical to the recently reported iFROG, however, the pulse shaper allows minimization of the required temporal samples through a judicious choice of the carrier envelope phase. By simply changing the transfer function, other measurement techniques were realized, such as an interferometric version of STRUT.

ACKNOWLEDGEMENTS This work was supported by NCCR Quantum Photonics (NCCR QP), research instrument of the Swiss National Science Foundation (SNSF).

\section{REFERENCES}

1 R. Trebino, Frequency Resolved Optical Gating (Springer, New York, 2002)

2 C. Iaconis, I.A. Walmsley, Opt. Lett. 23, 792 (1998)

3 J.L.A. Chilla, O.E. Martinez, Opt. Lett. 16, 39 (1991)

4 A.M. Weiner, Rev. Sci. Instrum. 71, 1929 (2000)

5 T. Brixner, G. Gerber, Opt. Lett. 26, 557 (2001)

6 J.C. Vaughan, T. Feurer, K.A. Nelson, Opt. Lett. 28, 2408 (2003)

7 B. Xu, J.M. Gunn, J.M. Dela Cruz, V.V. Lozovoy, M. Dantus, J. Opt. Soc. Am. B 23, 750 (2006)

8 B. von Vacano, T. Buckup, M. Motzkus, Opt. Lett. 31, 1154 (2006)

9 A. Monmayrant, M. Joffre, T. Oksenhendler, R. Herzog, D. Kaplan, P. Tournois, Opt. Lett. 28, 278 (2003)

10 T. Feurer, A. Glass, R. Sauerbrey, Appl. Phys. B 65, 295 (1997)

11 J.C. Vaughan, T. Feurer, K.W. Stone, K.A. Nelson, Opt. Express 14, 1314 (2006)

12 G. Stibenz, G. Steinmeyer, Opt. Express 13, 2617 (2005)

13 I.A. Roldán, I.G. Cormack, P.L. Alvarez, E.J. Gualda, D. Artigas, Opt. Express 12, 1169 (2004) 